\title{
Pollen Spectrum of Some Honey Samples Produced in Siirt-Turkey
}

\section{Siirt-Türkiye'de Üretilmiş Bazı Balların Polen Çeşitliliği}

\author{
Semra Gürbüz ${ }^{1 \oplus}$, Çiğdem Özenirler $^{2,3^{*} \oplus}$, Nazlı Mayda $^{2}$, Ömür Gençay Çelemli' ${ }^{2,3}$, Aslı Özkök $^{3 \odot}$ \\ ${ }^{1}$ Mardin Artuklu University, Tourism Faculty, Gastronomy and Culinary Art, Mardin, Turkey. \\ 2Hacettepe University, Faculty of Science, Department of Biology, Beytepe Ankara, Turkey. \\ ${ }^{3}$ Hacettepe University, Bee and Bee Products Research and Application Center, Beytepe, Ankara, Turkey.
}

\section{ABSTRACT}

$\mathrm{M}$ elissopalynology is the microscopic analysis of the pollen in the honey. This branch of palynology has been extensively used to determine the purity and quality of honey with its geographical and floral origins. The foraging plants of honeybees can be determined by analyzing the honey. In this study, the honeys originated from Siirt region were investigated. Totally 24 honey samples were evaluated with their total pollen numbers, moisture and pollen compositions. Total pollen numbers were ranged between 2086-55710, with the mean value of 22506. The pollen composition can be defined as relatively rich. It was found that $75 \%$ of the honeys were containing more than 10 plant taxa. Frequently observed plant families were Asteraceae, Fabaceae and Lamiaceae. According to these results we can claim that the examined regions of the city are really important for quality honey production.

\section{Key Words}

Honey, melissopalynology, pollen analysis, Siirt.

\section{öz}

aldaki polenin mikroskobik analizi melissopalinoloji olarak adlandırılmaktadır. Palinolojinin bu dalı, balın coğrafik kö kenini, saflığını ve kalitesini belirlemek için yoğun şekilde kullanılmaktadır. Bal arılarının nektar ve polen topladıkları bitkiler, balın mikroskobik analizi ile belirlenebilmektedir. Bu çalışmada, Siirt bölgesinden toplanan ballar incelenmiştir. 24 bal örneğinin toplam polen sayısı, nem oranı ve polen bileşimleri değerlendirilmiştir. Toplam polen sayısı, 2086-55710 arasında değişmekte oluo ortalama değeri 22506 'dır. Analizi yapılan bal örneklerinin polen bileşimi, nispeten zengin olarak tanımlanmıştır. Balların \%75'inin 10'dan fazla bitki taksonu içerdiği tespit edilmiştir. Bal örneklerinin mikroskobik analizi sonucu, sık gözlenen bitki aileleri Asteraceae, Fabaceae ve Lamiaceae olarak belirlenmiştir. Bu sonuçlara göre, kentin incelenen bölgelerinin zengin polen kaynaklı bal üretimi için önemli olduğunu iddia edebiliriz.

\section{Anahtar Kelimeler}

Bal, melissopalinoloji, polen analizi, Siirt.

Article History: Received: Feb 25, 2019; Revised: Mar 15, 2019; Accepted: May 27, 2019; Available Online: Sep 15, 2019 DOI: https://doi.org/10.15671/hjbc.532279

Correspondence to: Ç. Özenirler, Hacettepe University, Faculty of Science, Department of Biology, Beytepe, Ankara, Turkey; Hacettepe University, Bee and Bee Products Research and Application Center, Beytepe, Ankara, Turkey.

E-Mail: cozenir@hacettepe.edu.tr 


\section{INTRODUCTION}

ru urkey, is a large country with an area of $814.578 \mathrm{~km} 2$ and serving as a bridge between Asia and Europe. It has 81 city and all of them have different type climate and rich flora because of three phytogeographic (Mediterranean, Europe-Siberia and Irano-Turanian) regions [1]. Euro-Siberian region is divided into two provinces. One is euxine and the second one is hyrcanian. The Euxine province is larger than hyrcanian province in Turkey and it extends throughout northern Turkey. It consists deciduous forest and conifers. Mediterranean region includes South Anatolia, West Anatolia and Gelibolu peninsula. Irano-Turanian region includes Inner Anatolia and East Anatolia. It divides into two major vegetational areas. One is forested area and the other one is steppe $[1,2]$.

There are about 12000 plant species with 3000 endemics in Turkey. Up to 500 plant species are nectary plants used by honey bees [3]. Turkey is the second country in the world after China, with presence of bee colonies (7.8 million bee colony) and annual honey production (114 thousand tons) $[4,5]$.

Siirt is one of the 81 provinces in Turkey and it is located in Southeast Anatolia of Turkey (Figure 1) and it is the one of the important point in terms of organic honey. Republic of Turkey Ministry of Agriculture and Forestry and Turkish Statistical Institute (TUIK) have been reported that there are 1793 beehives and $1878 \mathrm{~kg}$ of organic honey production in Siirt region [6-9]. The terrestrial climate is dominant in the Siirt region and phytogeographical regions type is Irano-Turanian [10]. So generally steppe vegetation is common in this area [2].

In this study, the honeys originated from Siirt were investigated. Totally 24 honey samples were evaluated with their total pollen number and the pollen composition. Total polen number in ten gram honey (TPN10) and plant diversity were found with melissopalynological analyses.

\section{MATERIALS and METHODS}

\section{Collection of Samples}

Total 24 honey samples were collected from Siirt region in 2018 (Figure 2). Honey samples were brought to Hacettepe University, Palynology and Bee Products Analysis Laboratory for melissopalynological analysis.

\section{Melissopalynological Analysis}

The total number of pollen in honey gives information about honey quality. For this purpose, 10 grams of honey is weighed in a tube and distilled water is placed on it. Also one Lycopodium spore tablet, which is contain 9666 Lycopodium spores, is put in honey and water mix. This mix is heated until spore tablet melt and 10 drops liquid basic fuchsin added. After heating procedure mixture is centrifuged at 3500 rpm for 45 minutes. Then the top of the tube is poured and preparations are prepared from the bottom part with $50 \%$ glycerin water

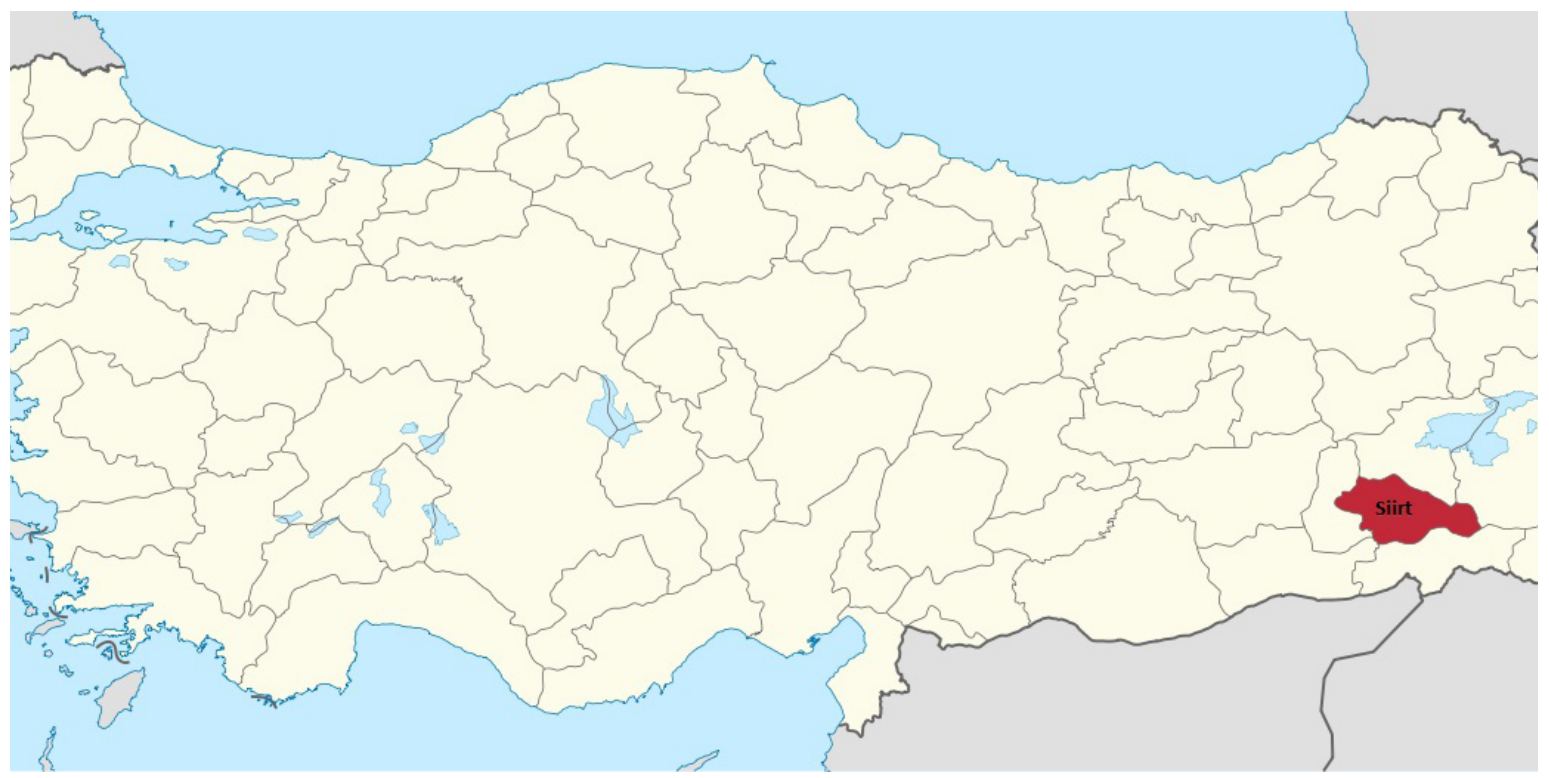

Figure 1. Siirt region in Turkey (This map was created to work with the ArcGis 10.2) [11]. 


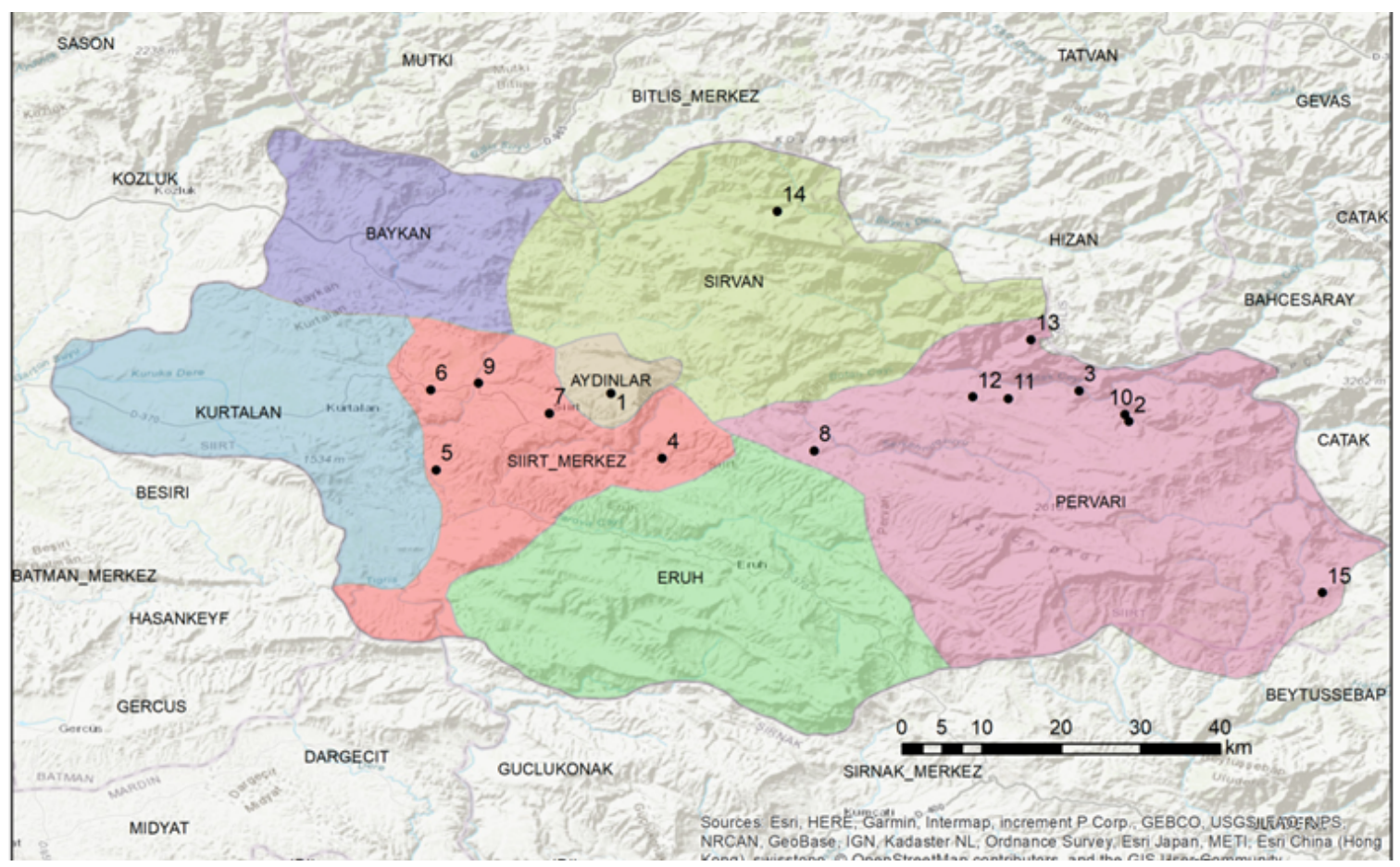

Figure 2. Siirt region in Turkey (This map was created to work with the ArcGis 10.2) [11].

mix. After that pollen and Lycopodium spore numbers are counted under microscope and calculated total pollen number (TPN10).

Beside total pollen number we determined plant diversity of the honey samples. For this purpose, 10 grams of honey is weighed in a tube and distilled water is placed on it. This mix is heated until honey melt. After that mixture is centrifuged at $3500 \mathrm{rpm}$ for 45 minutes. Then the top of the tube is poured and preparations are prepared from the bottom part with glycerin gelatin mix. One hour later preparations can be examined. The materials were prepared for examination under the microscope according to the method of Louveaux, 1978 and Sorkun, $2008[3,12]$.

\section{RESULTS and DISCUSSION}

Total 24 honey samples were investigated with mellissopalynological analysis in Siirt region. Total pollen number in ten gram honey (TPN10) was ranged between 2086-55710, with the mean value of 22506. Total pollen number between 20 thousand and 100 thousand honey is defined as good honey quality, between 100 thousand and 500 thousand is defined as rich honey quality $[3,13]$. In our study we found 9 of the 24 honeys are bigger than 20.000 (Table 1). Beside this, plant diversity is also important for determining honey origin. Pollen families are classified as dominant (D) ( $\geq 45 \%)$, secondary (S) (16-44\%), minor (M) (3-15\%), and trace (T) $(<3 \%)$ in the honey [14]. If the pollen of the plant taxon represented in the honey is more than $45 \%$, honey usually takes the name of that plant taxon $[3,15]$. In this study Onobrychis spp., Fabaceae, Salix spp., Centaurea spp., Carduus spp., Lamiaceae, Astragalus spp., plant taxa's pollen number was found as dominant (sample numbers: $4,6,8,10,11,12,14,23$ ) (Table 1). So honey samples can named with these taxa names. On the other hand, pollen taxa amounts are seconder, minor and trace percentage in other honey samples and these honey types are called mixed flower honey (sample numbers: 1, 2, 3, 5, 7, 9, 13, 15, 16, 17, 18, 19, 20, 21, 22, 24) (Table 1).

Our study is the first study which is analyzed microscopic analysis of Siirt region honey samples. Erez (2015) studied also microscopic analysis of the Siirt honeys. But they only examined the honeys of the Pervari region, the town of Siirt [16]. They found Asteraceae, Apiaceae, Lamiaceae, Malvaceae, Rosaceae commonly in Pervari region. In our study we found similar families (Fabaceae, Asteraceae and Lamiaceae) commonly in Siirt region (Table 1). 
Table 1. Siirt honey samples microscopic analysis results.

\begin{tabular}{|c|c|c|c|c|c|c|}
\hline $\begin{array}{l}\text { Sample } \\
\text { No }\end{array}$ & $\begin{array}{l}\text { Total } \\
\text { Pollen } \\
\text { Number } \\
\text { (TPN) }\end{array}$ & Plant Profile in Honey & $\begin{array}{c}\text { Pollen } \\
\text { Percentage } \\
(\%)^{*}\end{array}$ & Province & $\begin{array}{l}\text { Family } \\
\text { Number }\end{array}$ & $\begin{array}{l}\text { Taxa } \\
\text { Number }\end{array}$ \\
\hline 1 & 7837 & $\begin{array}{l}\text { Centaurea spp. } \\
\text { Fabaceae } \\
\text { Onobrychis spp. } \\
\text { Plantago spp. } \\
\text { Xanthium spp. } \\
\text { Poaceae } \\
\text { Asteracea }\end{array}$ & $\begin{array}{c}35.7(\mathrm{~S}) \\
21.4(\mathrm{~S}) \\
14.2(\mathrm{M}) \\
7.1(\mathrm{M}) \\
7.1(\mathrm{M}) \\
7.1(\mathrm{M}) \\
7.1(\mathrm{M})\end{array}$ & $\begin{array}{c}\text { Siirt } \\
\text { Pervari-Göl }\end{array}$ & 4 & 7 \\
\hline 2 & 44034 & $\begin{array}{l}\text { Carduus spp. } \\
\text { Centaurea spp. } \\
\text { Fabaceae } \\
\text { Liliaceae } \\
\text { Onobrychis spp. } \\
\text { Brassicaceae } \\
\text { Apiaceae } \\
\text { Rosaceae } \\
\text { Poaceae } \\
\text { Plantago spp. } \\
\text { Taraxacum spp. } \\
\text { Cephalaria spp. } \\
\text { Lamiaceae } \\
\text { Helianthus spp }\end{array}$ & $\begin{array}{c}32.6(\mathrm{~S}) \\
25(\mathrm{~S}) \\
11.5(\mathrm{M}) \\
7.3(\mathrm{M}) \\
6.1(\mathrm{M}) \\
5(\mathrm{M}) \\
4.6(\mathrm{M}) \\
3(\mathrm{M}) \\
1.5(\mathrm{~T}) \\
1.1(\mathrm{~T}) \\
0.76(\mathrm{~T}) \\
0.38(\mathrm{~T}) \\
0.38(\mathrm{~T}) \\
0.38(\mathrm{~T})\end{array}$ & $\begin{array}{c}\text { Siirt } \\
\text { Pervari- } \\
\text { Yukarıbalcılar }\end{array}$ & 10 & 14 \\
\hline 3 & 34935 & $\begin{array}{l}\text { Trifolium spp. } \\
\text { Apiaceae } \\
\text { Brassicaceae } \\
\text { Taraxacum spp. } \\
\text { Carduus spp. } \\
\text { Papaveraceae } \\
\text { Sanguisorba spp. } \\
\text { Lamiaceae } \\
\text { Salix spp. } \\
\text { Rosaceae } \\
\text { Scabiosa spp. } \\
\text { Echium spp. } \\
\text { Epilobium spp. } \\
\text { Thymus spp. } \\
\text { Onobrychis spp. } \\
\text { Centaurea spp. }\end{array}$ & $\begin{array}{c}29.7(\mathrm{~S}) \\
21.7(\mathrm{~S}) \\
13.1(\mathrm{M}) \\
8.5(\mathrm{M}) \\
5.7(\mathrm{M}) \\
4.5(\mathrm{M}) \\
4(\mathrm{M}) \\
2.8(\mathrm{~T}) \\
2.8(\mathrm{~T}) \\
2.2(\mathrm{~T}) \\
1.7(\mathrm{~T}) \\
0.5(\mathrm{~T}) \\
0.5(\mathrm{~T}) \\
0.5(\mathrm{~T}) \\
0.5(\mathrm{~T}) \\
0.5(\mathrm{~T})\end{array}$ & $\begin{array}{c}\text { Siirt } \\
\text { Pervari- } \\
\text { Yukarıbalcılar }\end{array}$ & 12 & 16 \\
\hline 4 & 5854 & $\begin{array}{l}\text { Onobrychis spp. } \\
\text { Fabaceae } \\
\text { Lamiaceae } \\
\text { Thymus spp. } \\
\text { Centaurea spp. } \\
\text { Apiaceae } \\
\text { Hedysarum spp. } \\
\text { Rosaceae }\end{array}$ & $\begin{array}{c}53.3(\mathrm{D}) \\
40(\mathrm{~S}) \\
31.1(\mathrm{~S}) \\
4.4(\mathrm{M}) \\
4.4(\mathrm{M}) \\
2.2(\mathrm{~T}) \\
2.2(\mathrm{~T}) \\
2.2(\mathrm{~T})\end{array}$ & $\begin{array}{c}\text { Siirt } \\
\text { Pervari- } \\
\text { Yukarıbalcılar }\end{array}$ & 5 & 8 \\
\hline 5 & 46873 & $\begin{array}{l}\text { Fabaceae } \\
\text { Liliaceae } \\
\text { Centaurea spp. } \\
\text { Carduus spp. } \\
\text { Plantago spp. } \\
\text { Berberidaceae } \\
\text { Brassicaceae } \\
\text { Apiaceae } \\
\text { Sanguisorba spp. } \\
\text { Taraxacum spp. }\end{array}$ & $\begin{array}{c}26(\mathrm{~S}) \\
19.1(\mathrm{~S}) \\
16.9(\mathrm{~S}) \\
13.4(\mathrm{M}) \\
13(\mathrm{M}) \\
7.8(\mathrm{M}) \\
1.3(\mathrm{~T}) \\
0.86(\mathrm{~T}) \\
0.86(\mathrm{~T}) \\
0.43(\mathrm{~T})\end{array}$ & $\begin{array}{c}\text { Siirt } \\
\text { Pervari-Çavuşlu }\end{array}$ & 8 & 10 \\
\hline
\end{tabular}


Table 1. Siirt honey samples microscopic analysis results. Continue

\begin{tabular}{|c|c|c|c|c|c|c|}
\hline $\begin{array}{l}\text { Sample } \\
\text { No }\end{array}$ & $\begin{array}{c}\text { Total } \\
\text { Pollen } \\
\text { Number } \\
\text { (TPN) }\end{array}$ & Plant Profile in Honey & $\begin{array}{c}\text { Pollen } \\
\text { Percentage } \\
(\%)^{*}\end{array}$ & Province & $\begin{array}{l}\text { Family } \\
\text { Number }\end{array}$ & $\begin{array}{c}\text { Taxa } \\
\text { Number }\end{array}$ \\
\hline 6 & 19488 & $\begin{array}{c}\text { Fabaceae } \\
\text { Asteraceae } \\
\text { Carduus spp. } \\
\text { Taraxacum spp. } \\
\text { Apiaceae } \\
\text { Echinops spp. } \\
\text { Lamiaceae } \\
\text { Cucurbitaceae } \\
\text { Geraniaceae }\end{array}$ & $\begin{array}{c}46(\mathrm{D}) \\
20(\mathrm{~S}) \\
10.6(\mathrm{M}) \\
9.3(\mathrm{M}) \\
4(\mathrm{M}) \\
3.3(\mathrm{M}) \\
3.3(\mathrm{M}) \\
2.6(\mathrm{~T}) \\
0.6(\mathrm{~T})\end{array}$ & $\begin{array}{c}\text { Siirt } \\
\text { Pervari-Güleçler }\end{array}$ & 6 & 9 \\
\hline 7 & 14793 & $\begin{array}{c}\text { Fabaceae } \\
\text { Asteraceae } \\
\text { Plantago spp. } \\
\text { Taraxacum spp. } \\
\text { Rumex spp. } \\
\text { Salix spp. } \\
\text { Berberidaceae } \\
\text { Apiaceae } \\
\text { Brassicaceae } \\
\text { Poaceae } \\
\text { Rosaceae }\end{array}$ & $\begin{array}{l}40.2(\mathrm{~S}) \\
34.7(\mathrm{~S}) \\
7.6(\mathrm{~S}) \\
5.4(\mathrm{M}) \\
3.2(\mathrm{M}) \\
2.1(\mathrm{~T}) \\
2.1(\mathrm{~T}) \\
1(\mathrm{~T}) \\
1(\mathrm{~T}) \\
1(\mathrm{~T}) \\
1(\mathrm{~T})\end{array}$ & $\begin{array}{c}\text { Siirt } \\
\text { Pervari-Sarıyaprak }\end{array}$ & 10 & 11 \\
\hline 8 & 14881 & $\begin{array}{l}\text { Salix spp. } \\
\text { Fabaceae } \\
\text { Apiaceae } \\
\text { Plantago spp. } \\
\text { Brassicaceae } \\
\text { Lamiaceae }\end{array}$ & $\begin{array}{c}50(\mathrm{D}) \\
19.2(\mathrm{~S}) \\
11.5(\mathrm{M}) \\
11.5(\mathrm{M}) \\
3.8(\mathrm{M}) \\
3.8(\mathrm{M})\end{array}$ & $\begin{array}{c}\text { Siirt-Şirvan } \\
\text { Kesmetaş }\end{array}$ & 6 & 6 \\
\hline 9 & 7072 & $\begin{array}{c}\text { Centaurea spp. } \\
\text { Onobrychis spp. } \\
\text { Liliaceae } \\
\text { Rosaceae } \\
\text { Brassicaceae } \\
\text { Trifolium spp. } \\
\text { Hedysarum spp. } \\
\text { Carduus spp. } \\
\text { Plantago spp. } \\
\text { Taraxacum spp. } \\
\text { Apiaceae } \\
\text { Teucrium spp. } \\
\text { Fabaceae } \\
\text { Papaveraceae } \\
\text { Lamiaceae }\end{array}$ & $\begin{array}{c}35.7(\mathrm{~S}) \\
11.2(\mathrm{M}) \\
7.1(\mathrm{M}) \\
7.1(\mathrm{M}) \\
7.1(\mathrm{M}) \\
6.1(\mathrm{M}) \\
5.1(\mathrm{M}) \\
5.1(\mathrm{M}) \\
4(\mathrm{M}) \\
3(\mathrm{M}) \\
2(\mathrm{~T}) \\
2(\mathrm{~T}) \\
2(\mathrm{~T}) \\
1(\mathrm{~T}) \\
1(\mathrm{~T})\end{array}$ & $\begin{array}{c}\text { Siirt } \\
\text { Pervari- } \\
\text { Yukarıbalcılar }\end{array}$ & 9 & 15 \\
\hline 10 & 9076 & $\begin{array}{c}\text { Centaurea spp. } \\
\text { Carduus spp. } \\
\text { Trifolium spp. } \\
\text { Brassicaceae } \\
\text { Onobrychis spp. } \\
\text { Papaveraceae } \\
\text { Taraxacum spp. } \\
\text { Rosaceae } \\
\text { Liliaceae } \\
\text { Fabaceae } \\
\text { Hedysarum spp. } \\
\text { Tilia spp. } \\
\text { Salix spp. } \\
\text { Plantago spp. } \\
\text { Teucrium spp. }\end{array}$ & $\begin{array}{l}53.7(\mathrm{D}) \\
9.43(\mathrm{M}) \\
7.5(\mathrm{M}) \\
6.6(\mathrm{M}) \\
5.6(\mathrm{M}) \\
4.7(\mathrm{M}) \\
2.8(\mathrm{~T}) \\
1.8(\mathrm{~T}) \\
1.8(\mathrm{~T}) \\
0.9(\mathrm{~T}) \\
0.9(\mathrm{~T}) \\
0.9(\mathrm{~T}) \\
0.9(\mathrm{~T}) \\
0.9(\mathrm{~T}) \\
0.9(\mathrm{~T})\end{array}$ & $\begin{array}{c}\text { Siirt } \\
\text { Merkez }\end{array}$ & 10 & 15 \\
\hline
\end{tabular}


300 Ç. Özenirler et al. / Hacettepe J. Biol. \& Chem., 2019, 47 (3), 295-303

Table 1. Siirt honey samples microscopic analysis results. Continue

\begin{tabular}{|c|c|c|c|c|c|c|}
\hline $\begin{array}{l}\text { Sample } \\
\text { No }\end{array}$ & $\begin{array}{l}\text { Total } \\
\text { Pollen } \\
\text { Number } \\
\text { (TPN) }\end{array}$ & Plant Profile in Honey & $\begin{array}{c}\text { Pollen } \\
\text { Percentage } \\
(\%)^{*}\end{array}$ & Province & $\begin{array}{l}\text { Family } \\
\text { Number }\end{array}$ & $\begin{array}{l}\text { Taxa } \\
\text { Number }\end{array}$ \\
\hline 11 & 7444 & $\begin{array}{l}\text { Carduus spp. } \\
\text { Trifolium spp. } \\
\text { Apiaceae } \\
\text { Campanula spp. } \\
\text { Centaurea spp. } \\
\text { Rosaceae } \\
\text { Lamiaceae } \\
\text { Chenopodiaceae } \\
\text { Geraniaceae } \\
\text { Sanguisorba spp. } \\
\text { Papaveraceae } \\
\text { Scabiosa spp. } \\
\text { Echium spp. } \\
\text { Asteraceae } \\
\text { Plantago spp. }\end{array}$ & $\begin{array}{l}48.6(\mathrm{D}) \\
20.8(\mathrm{~S}) \\
8.6(\mathrm{M}) \\
3.4(\mathrm{M}) \\
2.6(\mathrm{~T}) \\
2.6(\mathrm{~T}) \\
2.6(\mathrm{~T}) \\
2.6(\mathrm{~T}) \\
1.7(\mathrm{~T}) \\
1.7(\mathrm{~T}) \\
0.8(\mathrm{~T}) \\
0.8(\mathrm{~T}) \\
0.8(\mathrm{~T}) \\
0.8(\mathrm{~T}) \\
0.8(\mathrm{~T})\end{array}$ & $\begin{array}{c}\text { Siirt } \\
\text { Pervari-Merkez }\end{array}$ & 13 & 15 \\
\hline 12 & 3295 & $\begin{array}{l}\text { Lamiaceae } \\
\text { Trifolium spp. } \\
\text { Hedysarum spp. } \\
\text { Carduus spp. } \\
\text { Onobrychis spp. } \\
\text { Echium spp. } \\
\text { Salix spp. } \\
\text { Papaveraceae } \\
\text { Centaurea spp. }\end{array}$ & $\begin{array}{l}55.1(\mathrm{D}) \\
10.3(\mathrm{M}) \\
10.3(\mathrm{M}) \\
6.8(\mathrm{M}) \\
3.4(\mathrm{M}) \\
3.4(\mathrm{M}) \\
3.4(\mathrm{M}) \\
3.4(\mathrm{M}) \\
3.4(\mathrm{M})\end{array}$ & $\begin{array}{c}\text { Siirt } \\
\text { Merkez-Aktaş }\end{array}$ & 6 & 9 \\
\hline 13 & 2086 & $\begin{array}{l}\text { Onobrychis spp. } \\
\text { Lamiaceae } \\
\text { Trifolium spp. } \\
\text { Centaurea spp. } \\
\text { Salix spp. } \\
\text { Carduus spp. } \\
\text { Hedysarum spp. } \\
\text { Cistaceae } \\
\text { Apiaceae }\end{array}$ & $\begin{array}{c}34.2(\mathrm{~S}) \\
31.4(\mathrm{~S}) \\
11.4(\mathrm{M}) \\
8.5(\mathrm{M}) \\
2.8(\mathrm{~T}) \\
2.8(\mathrm{~T}) \\
2.8(\mathrm{~T}) \\
2.8(\mathrm{~T}) \\
2.8(\mathrm{~T})\end{array}$ & $\begin{array}{c}\text { Siirt } \\
\text { Pervari-Tosuntarla }\end{array}$ & 6 & 9 \\
\hline 14 & 13808 & $\begin{array}{l}\text { Trifolium spp. } \\
\text { Carduus spp. } \\
\text { Plantago spp. } \\
\text { Apiaceae } \\
\text { Lamiaceae } \\
\text { Poaceae } \\
\text { Taraxacum spp. } \\
\text { Papaveraceae } \\
\text { Rumex spp. } \\
\text { Sanguisorba spp. } \\
\text { Cistaceae }\end{array}$ & $\begin{array}{l}45(D) \\
30(S) \\
7(\mathrm{M}) \\
6(\mathrm{M}) \\
2(\mathrm{~T}) \\
2(\mathrm{~T}) \\
2(\mathrm{~T}) \\
2(\mathrm{~T}) \\
2(T) \\
1(T) \\
1(T)\end{array}$ & $\begin{array}{c}\text { Siirt } \\
\text { Merkez-Kışlacık }\end{array}$ & 10 & 11 \\
\hline 15 & 55710 & $\begin{array}{l}\text { Astragalus spp. } \\
\text { Fabaceae } \\
\text { Rosaceae } \\
\text { Taraxacum spp. } \\
\text { Trifolium spp. } \\
\text { Lamiaceae } \\
\text { Carduus spp. } \\
\text { Liliaceae } \\
\text { Apiaceae } \\
\text { Tilia spp. } \\
\text { Campanula spp. } \\
\text { Plantago spp. } \\
\text { Centaurea spp. } \\
\text { Salix spp. } \\
\text { Cistaceae } \\
\text { Rumex spp. } \\
\text { Brassicaceae } \\
\text { Poaceae } \\
\text { Asteraceae }\end{array}$ & $\begin{array}{l}36.4(\mathrm{~S}) \\
19.8(\mathrm{~S}) \\
6.6(\mathrm{M}) \\
3.9(\mathrm{M}) \\
3.9(\mathrm{M}) \\
3.9(\mathrm{M}) \\
3.3(\mathrm{M}) \\
3.3(\mathrm{M}) \\
3.3(\mathrm{M}) \\
2.6(\mathrm{~T}) \\
2.6(\mathrm{~T}) \\
1.9(\mathrm{~T}) \\
1.9(\mathrm{~T}) \\
1.3(\mathrm{~T}) \\
1.3(\mathrm{~T}) \\
1.3(\mathrm{~T}) \\
0.6(\mathrm{~T}) \\
0.6(\mathrm{~T}) \\
0.6(\mathrm{~T})\end{array}$ & $\begin{array}{c}\text { Siirt } \\
\text { Pervari }\end{array}$ & 14 & 19 \\
\hline
\end{tabular}


301 | Ç. Özenirler et al. / Hacettepe J. Biol. \& Chem., 2019, 47 (3), 295-303

Table 1. Siirt honey samples microscopic analysis results. Continue

\begin{tabular}{|c|c|c|c|c|c|c|}
\hline $\begin{array}{c}\text { Sample } \\
\text { No }\end{array}$ & $\begin{array}{l}\text { Total } \\
\text { Pollen } \\
\text { Number } \\
\text { (TPN) }\end{array}$ & Plant Profile in Honey & $\begin{array}{c}\text { Pollen } \\
\text { Percentage } \\
(\%)^{*}\end{array}$ & Province & $\begin{array}{l}\text { Family } \\
\text { Number }\end{array}$ & $\begin{array}{c}\text { Taxa } \\
\text { Number }\end{array}$ \\
\hline 16 & 34490 & $\begin{array}{l}\text { Carduus spp. } \\
\text { Trifolium spp. } \\
\text { Onobrychis spp. } \\
\text { Rosaceae } \\
\text { Astragalus spp. } \\
\text { Trifolium pratense } \\
\text { Brassicaceae } \\
\text { Lamiaceae } \\
\text { Fabaceae } \\
\text { Salix spp. } \\
\text { Plantago spp. } \\
\text { Echium spp. } \\
\text { Centaurea spp. } \\
\text { Sanguisorba spp. } \\
\text { Taraxacum spp. } \\
\text { Scabiosa spp. }\end{array}$ & $\begin{array}{l}25.4(\mathrm{~S}) \\
14.4(\mathrm{M}) \\
9.3(\mathrm{M}) \\
9.3(\mathrm{M}) \\
9.3(\mathrm{M}) \\
6.7(\mathrm{M}) \\
5.9(\mathrm{M}) \\
5.9(\mathrm{M}) \\
2.5(\mathrm{~T}) \\
2.5(\mathrm{~T}) \\
2.5(\mathrm{~T}) \\
1.6(\mathrm{~T}) \\
1.6(\mathrm{~T}) \\
0.8(\mathrm{~T}) \\
0.8(\mathrm{~T}) \\
0.8(\mathrm{~T})\end{array}$ & $\begin{array}{c}\text { Siirt } \\
\text { Pervari- } \\
\text { Aşağıbalcılar }\end{array}$ & 10 & 16 \\
\hline 17 & 44967 & $\begin{array}{l}\text { Carduus spp. } \\
\text { Liliaceae } \\
\text { Plantago spp. } \\
\text { Berberidaceae } \\
\text { Brassicaceae } \\
\text { Lamiaceae } \\
\text { Trifolium spp. } \\
\text { Centaurea spp. } \\
\text { Astragalus spp. } \\
\text { Taraxacum spp. } \\
\text { Geraniaceae } \\
\text { Rumex spp. } \\
\text { Cephalaria spp. }\end{array}$ & $\begin{array}{c}17.6(\mathrm{~S}) \\
16.4(\mathrm{~S}) \\
12.9(\mathrm{M}) \\
10.5(\mathrm{M}) \\
9.4(\mathrm{M}) \\
9.4(\mathrm{M}) \\
8.2(\mathrm{M}) \\
7(\mathrm{M}) \\
3.5(\mathrm{M}) \\
1.17(\mathrm{~T}) \\
1.17(\mathrm{~T}) \\
1.17(\mathrm{~T}) \\
1.17(\mathrm{~T})\end{array}$ & $\begin{array}{c}\text { Siirt } \\
\text { Pervari-Güleçler }\end{array}$ & 10 & 13 \\
\hline 18 & 9502 & $\begin{array}{l}\text { Carduus spp. } \\
\text { Trifolium spp. } \\
\text { Cistaceae } \\
\text { Trifolium pratense } \\
\text { Brassicaceae } \\
\text { Salix spp. } \\
\text { Apiaceae } \\
\text { Tilia spp. } \\
\text { Poaceae } \\
\text { Plantago spp. } \\
\text { Lamiaceae } \\
\text { Taraxacum spp. } \\
\text { Liliaceae }\end{array}$ & $\begin{array}{c}22.2(\mathrm{~S}) \\
14.2(\mathrm{M}) \\
12.6(\mathrm{M}) \\
9.5(\mathrm{M}) \\
7.9(\mathrm{M}) \\
7.9(\mathrm{M}) \\
6.3(\mathrm{M}) \\
4.7(\mathrm{M}) \\
4.7(\mathrm{M}) \\
3.1(\mathrm{M}) \\
3.1(\mathrm{M}) \\
1.5(\mathrm{~T}) \\
1.5(\mathrm{~T})\end{array}$ & $\begin{array}{c}\text { Siirt } \\
\text { Merkez-Köprübaşı }\end{array}$ & 11 & 13 \\
\hline 19 & 12082 & $\begin{array}{c}\text { Salix spp. } \\
\text { Cistaceae } \\
\text { Trifolium pratense } \\
\text { Brassicaceae } \\
\text { Apiaceae } \\
\text { Trifolium spp. } \\
\text { Tilia spp. } \\
\text { Carduus spp. } \\
\text { Sanguisorba spp. } \\
\text { Poaceae } \\
\text { Lamiaceae } \\
\text { Rumex spp. } \\
\text { Taraxacum spp. }\end{array}$ & $\begin{array}{c}24.4(\mathrm{~S}) \\
12.2(\mathrm{M}) \\
10.2(\mathrm{M}) \\
10.2(\mathrm{M}) \\
8.1(\mathrm{M}) \\
6.1(\mathrm{M}) \\
6.1(\mathrm{M}) \\
6.1(\mathrm{M}) \\
4(\mathrm{M}) \\
4(\mathrm{M}) \\
4(\mathrm{M}) \\
2(\mathrm{~T}) \\
2(\mathrm{~T})\end{array}$ & $\begin{array}{c}\text { Siirt } \\
\text { Merkez-Bayraktepe }\end{array}$ & 11 & 13 \\
\hline
\end{tabular}


Table 1. Siirt honey samples microscopic analysis results. Continue

\begin{tabular}{|c|c|c|c|c|c|c|}
\hline $\begin{array}{l}\text { Sample } \\
\text { No }\end{array}$ & $\begin{array}{l}\text { Total } \\
\text { Pollen } \\
\text { Number } \\
\text { (TPN) }\end{array}$ & Plant Profile in Honey & $\begin{array}{c}\text { Pollen } \\
\text { Percentage } \\
(\%)^{*}\end{array}$ & Province & $\begin{array}{l}\text { Family } \\
\text { Number }\end{array}$ & $\begin{array}{l}\text { Taxa } \\
\text { Number }\end{array}$ \\
\hline 20 & 17249 & $\begin{array}{l}\text { Centaurea spp. } \\
\text { Carduus spp. } \\
\text { Taraxacum spp. } \\
\text { Rosaceae } \\
\text { Brassicaceae } \\
\text { Liliaceae } \\
\text { Plantago spp. } \\
\text { Apiaceae } \\
\text { Salix spp. } \\
\text { Onobrychis spp. } \\
\text { Papaveraceae } \\
\text { Cephalaria spp. } \\
\text { Lamiaceae } \\
\text { Sanguisorba spp. } \\
\text { Convolvulus spp. }\end{array}$ & $\begin{array}{c}31.7(\mathrm{~S}) \\
22.2(\mathrm{~S}) \\
12.6(\mathrm{M}) \\
6.3(\mathrm{M}) \\
6.3(\mathrm{M}) \\
5.5(\mathrm{M}) \\
3.9(\mathrm{M}) \\
3.1(\mathrm{M}) \\
3(\mathrm{M}) \\
1.6(\mathrm{~T}) \\
0.8(\mathrm{~T}) \\
0.8(\mathrm{~T}) \\
0.8(\mathrm{~T}) \\
0.8(\mathrm{~T}) \\
0.8(\mathrm{~T})\end{array}$ & $\begin{array}{c}\text { Siirt } \\
\text { Merkez-Bayraktepe }\end{array}$ & 13 & 15 \\
\hline 21 & 19596 & $\begin{array}{l}\text { Trifolium spp. } \\
\text { Carduus spp. } \\
\text { Astragalus spp. } \\
\text { Plantago spp. } \\
\text { Centaurea spp. } \\
\text { Lamiaceae } \\
\text { Cistaceae } \\
\text { Apiaceae } \\
\text { Geraniaceae } \\
\text { Papaveraceae } \\
\text { Taraxacum spp. } \\
\text { Onobrychis spp. } \\
\text { Rosaceae }\end{array}$ & $\begin{array}{l}41(\mathrm{~S}) \\
28(\mathrm{~S}) \\
7(\mathrm{M}) \\
6(\mathrm{M}) \\
5(\mathrm{M}) \\
3(\mathrm{M}) \\
3(\mathrm{M}) \\
2(\mathrm{~T}) \\
1(\mathrm{~T}) \\
1(\mathrm{~T}) \\
1(\mathrm{~T}) \\
1(\mathrm{~T}) \\
1(\mathrm{~T})\end{array}$ & $\begin{array}{c}\text { Siirt } \\
\text { Pervari-Güleçler }\end{array}$ & 9 & 13 \\
\hline 22 & 36169 & $\begin{array}{l}\text { Trifolium pratense } \\
\text { Apiaceae } \\
\text { Taraxacum spp. } \\
\text { Papaveraceae } \\
\text { Cistaceae } \\
\text { Brassicaceae } \\
\text { Lamiaceae } \\
\text { Carduus spp. } \\
\text { Astragalus spp. } \\
\text { Salix spp. } \\
\text { Rosaceae } \\
\text { Centaurea spp. } \\
\text { Scabiosa spp. } \\
\text { Poaceae } \\
\text { Sanguisorba spp. } \\
\text { Onobrychis spp. } \\
\text { Malvaceae }\end{array}$ & $\begin{array}{c}17.2(\mathrm{~S}) \\
13.2(\mathrm{M}) \\
11.9(\mathrm{M}) \\
11.9(\mathrm{M}) \\
11.2(\mathrm{M}) \\
9.9(\mathrm{M}) \\
7.2(\mathrm{M}) \\
5.2(\mathrm{M}) \\
3.3(\mathrm{M}) \\
1.9(\mathrm{~T}) \\
1.3(\mathrm{~T}) \\
1.3(\mathrm{~T}) \\
1.3(\mathrm{~T}) \\
0.66(\mathrm{~T}) \\
0.66(\mathrm{~T}) \\
0.66(\mathrm{~T}) \\
0.66(\mathrm{~T})\end{array}$ & $\begin{array}{c}\text { Siirt } \\
\text { Pervari-Kopik }\end{array}$ & 13 & 17 \\
\hline 23 & 26302 & $\begin{array}{l}\text { Astragalus spp. } \\
\text { Apiaceae } \\
\text { Trifolium spp. } \\
\text { Lamiaceae } \\
\text { Plantago spp. } \\
\text { Trifolium pratense } \\
\text { Rosaceae } \\
\text { Tilia spp. } \\
\text { Salix spp. } \\
\text { Brassicaceae } \\
\text { Poaceae } \\
\text { Campanula spp. }\end{array}$ & $\begin{array}{l}57.3(\mathrm{D}) \\
9.8(\mathrm{M}) \\
8.2(\mathrm{M}) \\
8.2(\mathrm{M}) \\
4.9(\mathrm{M}) \\
1.6(\mathrm{~T}) \\
1.6(\mathrm{~T}) \\
1.6(\mathrm{~T}) \\
1.6(\mathrm{~T}) \\
1.6(\mathrm{~T}) \\
1.6(\mathrm{~T}) \\
1.6(\mathrm{~T})\end{array}$ & $\begin{array}{c}\text { Siirt } \\
\text { Pervari- } \\
\text { Yukarıbalcılar }\end{array}$ & 10 & 12 \\
\hline
\end{tabular}


Table 1. Siirt honey samples microscopic analysis results. Continue

\begin{tabular}{|c|c|c|c|c|c|c|}
\hline $\begin{array}{c}\text { Sample } \\
\text { No }\end{array}$ & $\begin{array}{l}\text { Total } \\
\text { Pollen } \\
\text { Number } \\
\text { (TPN) }\end{array}$ & Plant Profile in Honey & $\begin{array}{c}\text { Pollen } \\
\text { Percentage } \\
(\%)^{*}\end{array}$ & Province & $\begin{array}{l}\text { Family } \\
\text { Number }\end{array}$ & $\begin{array}{c}\text { Taxa } \\
\text { Number }\end{array}$ \\
\hline 24 & 52601 & $\begin{array}{c}\text { Astragalus spp. } \\
\text { Apiaceae } \\
\text { Rosaceae } \\
\text { Trifolium pratense } \\
\text { Plantago spp. } \\
\text { Liliaceae } \\
\text { Brassicaceae } \\
\text { Cistaceae } \\
\text { Campanula spp. } \\
\text { Taraxacum spp. } \\
\text { Carduus spp. } \\
\text { Tilia spp. } \\
\text { Lamiaceae } \\
\text { Caryophyllaceae } \\
\text { Papaveraceae } \\
\text { Cephalaria spp. } \\
\text { Scabiosa spp. }\end{array}$ & $\begin{array}{c}40(\mathrm{~S}) \\
16.8(\mathrm{~S}) \\
6.4(\mathrm{M}) \\
5.6(\mathrm{M}) \\
4(\mathrm{M}) \\
3.2(\mathrm{M}) \\
3.2(\mathrm{M}) \\
3.2(\mathrm{M}) \\
3.2(\mathrm{M}) \\
2.4(\mathrm{~T}) \\
2.4(\mathrm{~T}) \\
2.4(\mathrm{~T}) \\
2.4(\mathrm{~T}) \\
1.6(\mathrm{~T}) \\
1.6(\mathrm{~T}) \\
0.8(\mathrm{~T}) \\
0.8(\mathrm{~T})\end{array}$ & $\begin{array}{c}\text { Siirt } \\
\text { Pervari- } \\
\text { Yukarıbalcılar }\end{array}$ & 14 & 17 \\
\hline
\end{tabular}

* $\geq \% 45$ Dominant (D), (\%16-44) Seconder (S), (\%3-15) Minor (M), (<\%3) Trace (T).

The pollen composition can be defined as relatively rich. It was found that $75 \%$ of the honeys were containing more than 10 plant taxa. It was found that dominantly observed plant taxa were Onobrychis spp., Fabaceae, Salix spp., Centaurea spp., Carduus spp., Lamiaceae, Astragalus spp. According to these results, we can claim that the examined regions of the city are important honey production areas. It should be emphasized that these plants are important for apiculture in the region and care should be taken not to use them as grassland in flowering periods.

\section{Acknowledgments}

This research was supported by Southeastern Anatolia Project Regional Development Administration within the scope of the 2015A020020 Southeastern Anatolia Organic Agriculture Cluster Project.

\section{References}

1. M. Avcl, M., Türkiye'nin flora bölgeleri ve Anadolu Diagonali'ne coğrafi bir yaklaşım. Türk Coğ. Der., 28 (1993) 225-248.

2. P. H. Davis, Flora of Turkey. Flora of Turkey., 1965.

3. K. Sorkun, Turkey's Nectarious Plants, Pollen and Honey. 2008, Palme Press, Ankara.

4. FAO, Food and Agriculture Organization of the United Nations. (cited 2019; Available from: http://www.fao.org/ faostat/en/\#data/QL.

5. TAB, Turkish Association of Beekeepers. 2019; Available from: http://www.tab.org.tr/.
6. Republic of Turkey Ministry of Agriculture and Forestry. [cited 2019; Available from: www.tarim.gov.tr.

7. A. Kiracı, M. Çelik, Y. Birinci, S. S. Aktuğ, Siirt İlinin Sosyal ve Ekonomik Yapısı. İkt. Yen. Der. 2 (2015) 17-55.

8. Ç. Oğuzhan, E. Karakaya, Siirt II Merkezindeki Tüketicilerin Organik Ürün Tüketim Tercihleri ve Tercihlerini Etkileyen Faktörlerin Belirlenmesi. Adnan Menderes Üniversitesi Zir. Fak. Der. 15 (2018) 33-41.

9. TUIK. Turkish Statistical Institute. Organik Bitkisel Üretim, cited 2019; Available from: www.tuik.gov.tr.

10. Y. Muratgeldiev, M. Küçüködük, Ü. Bingöl, K. Güney, F. Geven, İran-Turan Floristik Bölgesi. Selçuk Üniversitesi Fen Fak. Fen Der., 2000. 1(2000) 119-124.

11. ArcGIS. 10.2. Available from: arcgis.hacettepe.edu.tr.

12. J. Louveaux, A. Maurizio, G. Vorwohl, Methods of Melissopalynology. Bee World, 59 (1978) 139-157.

13. M.J. Feller-Demalsy, J. Parent, A.A. Strachan, Microscopic analysis of honeys from Manitoba, Canada. J. Api. Res., 28 (1989) 41-49.

14. F. Corvucci, L. Nobili, D. Mellucci, V.T. Grillenzoni, The discrimination of honey origin using melissopalynology and Raman spectroscopy techniques coupled with multivariate analysis. Food Chem., 169 (2015) 297-304.

15. L. F. Cuevas-Glory, J. A. Pino, L. S. Santiago, E. Sauri-Duch, A review of volatile analytical methods for determining the botanical origin of honey. Food Chem., 103 (2007) 10321043.

16. M. E. Erez, O. Karabacak, L. Kaycı, M. Fidan, Y. Kaya, Characterization of Multifloral Honeys of Pervari Region with Different Properties. Türkiye Tarımsal Araştırmalar Dergisi, 2 (2015) 40-46. 